\title{
Avaliação Heurística de Interfaces de Sistemas Gamificados no Contexto Educacional: um processo e lições aprendidas
}

\author{
João Carlos Lima e Silva ${ }^{1}$, \\ Flávio Eduardo Aoki Horita ${ }^{1}$, Carla Lopes Rodriguez ${ }^{1}$, Rafaela Vilela da Rocha ${ }^{1}$ \\ ${ }^{1} \mathrm{CMCC}$ - Universidade Federal do ABC (UFABC) \\ Santo André - SP - Brazil \\ \{joao.e, flavio.horita, c.rodriguez, rafaela.rocha,\}@ufabc.edu.br
}

\begin{abstract}
The use of gamification as a strategy to engage students has proved to be very efficient. However, the results can be lost if the system does not present good usability. In this context, a process was proposed to heuristically evaluate the usability of learning gamification systems, in order to find strengths and weaknesses of the interfaces in some features. It can be observed that the main functionalities of these systems presented good usability, but functionalities like "Account Creation" have faltered in essential points of usability, compromising the experience of the users. The suggested process had good results in identifying the strengths and weaknesses of the applications.
\end{abstract}

Resumo. O uso da gamificação em sistemas educacionais tem se mostrado eficiente como estratégia para engajar os alunos. Porém, os resultados podem ser perdidos se o sistema não apresentar uma boa usabilidade. Nesse contexto, foi proposto e aplicado um processo para avaliar heurísticamente a usabilidade de sistemas educacionais gamificados com o objetivo de identificar pontos positivos e negativos das interfaces. Pode-se observar que as funcionalidades principais desses sistemas apresentaram uma boa usabilidade, porém funcionalidades como "Criação de Conta" falharam em pontos essenciais da usabilidade, comprometendo a experiência dos usuários. O processo proposto mostrou bons resultados na identificação dos pontos fortes e fracos das aplicações.

\section{Introdução}

O interesse pelo tema da gamificação vem crescendo em ritmo acelerado [Kapp 2012]. Essa técnica tem sido usada para melhorar a motivação dos alunos durante e fora das aulas. Gamificação consiste em utilizar elementos de jogos em outros ambientes [Alves 2014]. Exemplos desses elementos são: pontos, níveis, ranking, missões e medalhas.

O apelo visual dos ambientes gamificados tem papel importante no sucesso de um sistema gamificado [Mattar 2010]. Falhas na interação, informações confusas, erros pouco significativos e dificuldade em acessar funcionalidades podem colocar a perder os resultados obtidos pela gamificação.

No contexto educacional esses riscos são ainda maiores. Uma teoria bastante difundida nesse contexto é a teoria do flow, que incentiva o equilíbrio entre o desafio e a habilidade do aluno de modo a manter o aprendizado em um fluxo crescente [Csikszentmihalyi 2008]. Quando o aluno está em flow ele tem uma percepção diferente 
VIII Congresso Brasileiro de Informática na Educação (CBIE 2019)

Anais do XXX Simpósio Brasileiro de Informática na Educação (SBIE 2019)

da passagem do tempo e o aprendizado acontece de forma natural e prazerosa. Uma interface pouco amigável ou com falhas severas afasta o aluno dessa sensação, comprometendo a experiência [Kiili et al. 2012].

Para melhor compreensão da qualidade das interfaces das soluções educacionais gamificadas existentes, cabe uma análise dos pontos positivos e negativos com a finalidade de apresentar boas práticas para a construção de interfaces gráficas de usuários em ambientes gamificados.

Neste contexto, este trabalho apresenta e utiliza um processo de avaliação de interfaces de sistemas gamificados no contexto educacional baseado em técnicas de avaliação heurística. O processo é composto por três passos: 1) avaliar a usabilidade de forma heurística 2) listar os pontos fortes da aplicação e avaliar qualitativamente 3) listar os pontos fracos da aplicação e avaliar qualitativamente.

Na Seção 2, é apresentado o referencial teórico de gamificação e avaliação heurística. Na Seção 3 são apresentadas publicações que relacionadas à presente pesquisa. Na Seção 4 é apresentado o processo utilizado para avaliar as interfaces. Na Seção 5 são apresentados e discutidos os resultados da avaliação realizada. Finalmente, na Seção 6 são apresentadas as conclusões da avaliação e as recomendações de trabalhos futuros.

\section{Referencial Teórico}

\subsection{Gamificação}

O termo gamificação (gamification) significa utilização de elementos de jogos, (pontuação, missões, ranking, etc) em ambientes que não são de jogos [Kapp 2012]. Existe um grande número de aplicações da gamificação nas áreas de publicidade, saúde e educação. Esse fenômeno pode ser explicado, principalmente, pelo potencial da gamificação para influenciar, engajar e motivar pessoas [Alves 2014].

Entre as principais vantagens do uso da gamificação na educação, podemos destacar o retorno imediato de acertos e erros, informação sob demanda, auto-regulação da aprendizagem, trabalho em equipe e aprendizagem colaborativa [Domingues et al. 2013]. Alguns exemplos de uso de gamificação na educação são Duolingo, Geekie Game e ClassDojo. Esses sistemas apresentam características e públicos diferentes, porém os três possuem um forte apelo visual.

Para o sucesso de uma aplicação gamificada é muito importante que as interações entre o usuário e o sistema apresentem robustez e qualidade [Marache-Francisco and Brangier 2013]. Para atingir esse nível de qualidade é necessário criar soluções de gamificação centradas na experiência do usuário, tendo em vista sempre a maneira com que o usuário interage com o sistema, dando a ele liberdade de escolha e prevenindo erros [Botha et al. 2014].

Segundo [Domingues et al. 2013] um dos principais motivos para o baixo envolvimento dos alunos com sistemas gamificados é a complexidade de interações com os sistemas. Essas dificuldades podem ser minimizadas através de um bom projeto de interface de usuário.

\subsection{Avaliação Heurística}

Avaliação Heurística é um método informal de análise de usabilidade realizado por meio da observação da interface durante a sua utilização [Nielsen 1994]. Por ser um método 
VIII Congresso Brasileiro de Informática na Educação (CBIE 2019)

Anais do XXX Simpósio Brasileiro de Informática na Educação (SBIE 2019)

mais rápido de ser aplicado, tem sido uma das principais formas de avaliar a qualidade de interfaces de usuário [da Rocha and Baranauskas 2003].

Essa avaliação envolve um pequeno conjunto de avaliadores examinando a interface e julgando suas características de acordo com princípios reconhecidos de usabilidade, denominados heurísticas. De forma ideal, os avaliadores são especialistas em usabilidade e realizam algumas funcionalidades do sistema, pelo menos duas vezes, listando os pontos positivos e negativos de sua utilização [Nielsen and Landauer 1993].

A presente pesquisa realiza uma avaliação heurística baseada na lista revisada de [Nielsen and Landauer 1993] onde o número de avaliadores é uma variável importante para o bom resultado da avaliação. [Nielsen and Molich 1990] concluem a partir de uma pesquisa com 182 avaliações heurísticas, que o número ideal de avaliadores está entre três e cinco. O artefato da avaliação heurística é um relatório de avaliação que cita os pontos de falha e sua severidade relacionando com cada um dos tópicos avaliados [da Rocha and Baranauskas 2003].

Atualmente, existem outras heurísticas para avaliação de sistemas específicos. [Tondello et al. 2016] propõem a avaliação de design de jogos através de um conjunto de 28 heurísticas. Pelo fato dessas heurísticas serem específicas para jogos e não se adaptarem a gamificação, optou-se por utilizar as heurísticas clássicas de Jakob Nielsen.

\section{Trabalhos Relacionados}

Esta seção descreve brevemente estudos de avaliação heurísticas de aplicativos gamificados realizados por [Barbosa et al. 2014], [Souza and Souto 2015] e [Zancan 2018]. Esses trabalhos realizam pesquisas com o mesmo objetivos deste.

[Barbosa et al. 2014] realizaram três avaliações de interatividade em um mesmo aplicativo gamificado de saúde. Na pesquisa foram utilizadas Avaliação Heurística, Métodos de Inspeção Semiótica e Avaliação de Comunicabilidade. Além disso, o aplicativo foi avaliado em diversas plataformas e diversos dispositivos. Foram identificadas poucas falhas no aplicativo, com destaque para a diferença no design entre diferentes plataformas, mas no geral ele cumpre de modo satisfatório a proposta de motivar os usuários à prática de atividades físicas.

[Souza and Souto 2015] apresentam um estudo de viabilidade do uso de heurísticas de jogos para avaliar aplicativos gamificados. Os autores selecionaram 26 heurísticas baseadas em trabalhos relacionados e aplicaram na avaliação do aplicativo de ensino de línguas Duolingo. Após a análise da avaliação, concluiu-se que é viável a aplicação de heurísticas de jogos em aplicativos gamificados, porém sugere a criação de novas heurísticas específicas para essa finalidade com o objetivo de completar as já existentes.

[Zancan 2018] realizou a avaliação de aplicativo gamificado intitulado Quiz Fórmulas Químicas e de um jogo educacional chamado Coleta Seletiva, ambos voltados para o ensino de química. Após a avaliação verificou-se que os dois atendem os objetivos propostos de forma satisfatória, porém nesse estudo a avaliação não focou no caráter gamificado das aplicações.

De forma geral, os trabalhos apresentados sinalizam uma necessidade de um processo de avaliação de usabilidade focado em aplicativos gamificados. 
VIII Congresso Brasileiro de Informática na Educação (CBIE 2019)

Anais do XXX Simpósio Brasileiro de Informática na Educação (SBIE 2019)

\section{Metodologia}

Nesta seção são apresentados o processo proposto para a avaliação heurística de sistemas, os objetos de estudos utilizados para a validação do processos, uma breve descrição dos participantes e, finalmente os dados coletados.

\subsection{Processo de Avaliação Heurística}

Para atingir os objetivos da presente investigação foi desenhado um processo de avaliação de interfaces gráficas de aplicativos gamificados para aplicação educacional. O processo está ilustrado na Figura 1.

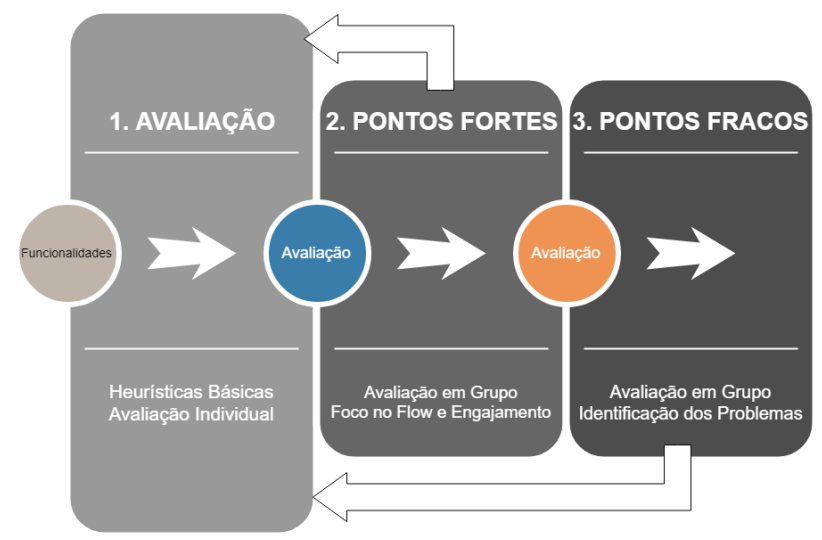

Figura 1. Processo de Avaliação Heurística de Sistemas Educacionais Gamifi-
cado

O primeiro passo do processo é aplicar a avaliação heurística atribuindo uma nota de um à cinco para cada uma das heurísticas clássicas de Nilsen. Preferencialmente, essa avaliação deve ser realizada por pelo menos três avaliadores com experiência nesse tipo de análise [Nielsen 1994]. Essa avaliação é conduzida de forma individual com cada avaliador realizando a funcionalidade no sistema. Nesse momento é importante que o avaliador tenha em mente as sensações que a gamificação proporciona, isto é, deve ser verificado se a experiência do flow é observada e se o uso do sistema promove o engajamento.

O segundo passo é a análise das funcionalidades que obtiveram a maior pontuação. Essa análise é realizada em grupo, com todos os avaliadores reunidos. O objetivo é realizar uma avaliação qualitativa que identifique as qualidade presentes na interação com essas funcionalidades.

No passo seguinte, uma análise similar é a realizada, no entanto, o foco consiste em debater as funcionalidades com as menores pontuações. Essa última avaliação é importante para identificar os pontos onde o engajamento e oflow são prejudicados por conta da usabilidade falha. Assim, o projeto pode ser corrigido ou as lições aprendidas podem ser utilizadas em novos projetos.

Durante os passos 2 e 3, caso haja divergência entre os avaliadores, ou seja identificados pontos que necessitem de uma reavaliação, pode-se realizar uma nova rodada de avaliação heurística em alguma funcionalidade específica, desta vez em conjunto. 


\subsection{Objetos de Estudo}

Após a definição do processo, foram avaliados heuristicamente três sistemas gamificados: Duolingo $^{1}$, Geekie Games ${ }^{2}$ e ClassDojo ${ }^{3}$. Os sistemas foram escolhidos por conveniência, baseado na disponibilidade de acesso gratuito e em sua aplicação educacional. Outros sistemas foram considerados para essa avaliação, porém não selecionados por serem pagos, entre os quais podemos citar Rosetta Stone ${ }^{4}$ e Feedback Panda ${ }^{5}$.

Os sistemas selecionados para a avaliação foram:

- Duolingo: aplicativo (web e mobile) de ensino de línguas que possui diversas avaliações positivas nas lojas de aplicativos. O usuário é desafiado a manter uma ofensiva de dias contínuos de estudo. Conforme realiza as atividades novas fases vão sendo disponibilizadas. O usuário é premiado com lingots conforme participa das atividades.

- Geekie Games: site voltado para a preparação de alunos para o Exame Nacional do Ensino Médio (ENEM). O participante assiste vídeos, tem acesso à textos e responde questionários. Essas atividades liberam novas etapas e são premiadas com bolts. O sistema sugere as atividades de acordo com o desempenho do aluno, para sanar lacunas de aprendizagem.

- ClassDojo: site e aplicativo para motivar os alunos a terem atitudes positivas. Também é possível a comunicação da escola com os responsáveis. Os alunos são premiados com o badge conforme evidenciam habilidades. Esses prêmios são acompanhados de pontos que podem ser visualizados pelos alunos e por seus responsáveis.

\subsection{Participantes}

Seguindo a recomendação de [Nielsen and Molich 1990], a avaliação foi realizada por três avaliadores de forma independente. Os avaliadores foram selecionados de acordo com a disponibilidade em participar da pesquisa e todos tem experiência de mais de dois anos em avaliação de usabilidade. Dois avaliadores possuem graduação em Ciência da Computação e um em Sistemas de Informação. Todos avaliadores são professores de ensino técnico de nível médio. Dois deles possuem especialização na área de desenvolvimento de software. Um deles também atua profissionalmente como gerente de projetos de software.

\subsection{Coleta dos Dados}

Durante a execução do processo metodológico com os participantes, cada um deles recebeu uma lista de cinco funcionalidades de cada sistema conforme Quadro 1. As funcionalidades foram selecionadas levando em consideração o fluxo principal do sistema, com foco naquelas de maior valor para o usuário, de acordo com o objetivo principal do sistema. Para cada uma das funcionalidades, eles deveriam atribuir uma nota de 1 a 5 , sendo 1 = péssimo, 2 = ruim, 3 = regular, 4 = bom e 5 = ótimo. Essas notas foram atribuídas para cada uma das dez heurísticas clássicas de Nielsen.

\footnotetext{
${ }^{1}$ https://www.duolingo.com

${ }^{2}$ https://geekiegames.geekie.com.br

${ }^{3} \mathrm{https}: / / \mathrm{www} . c l a s s d o j o . c o m$

${ }^{4}$ https://www.rosettastone.com/lp/sbsr/sitewide/

${ }^{5}$ https://www.feedbackpanda.com/
} 


\begin{tabular}{c|c|c} 
Duolingo & Geekie Game & ClassDojo \\
\hline Criar Conta & Criar Conta & Criar Conta \\
Tela Inicial & Plano de Estudo & Portifólio \\
Conquistas & Aula & Lista de Alunos \\
Atividades & O que + cai no ENEM & Criar Atividade \\
Configurar Conta & Desempenho & Badge
\end{tabular}

Quadro 1. Funcionalidades avaliadas por sistemas

Após a análise individual, os avaliadores se juntaram para consolidar as avaliações. Esse análise aconteceu presencialmente em uma sala de reuniões. As notas atribuídas foram discutidas e os principais pontos positivos e negativos foram listados para uma análise qualitativa mais aprofundada realizada pelos três avaliadores de forma conjunta. Em todos os critérios avaliados houve consenso entre os avaliadores.

\section{Resultados}

A consolidação das avaliações apresentou algumas deficiências nas interfaces avaliadas. Por outro lado, diversas estratégias se mostraram bastante eficientes em concordância com as recomendações de usabilidade.

\subsection{Pontos Negativos}

Nessa Seção serão apresentados os problemas encontrados. Na tela de cadastro de usuários do Geekie Games, o usuário só é avisado que o campo "celular" é obrigatório depois que o usuário clica em "cadastrar". Embora a mensagem de erro seja clara e ajude a corrigir o problema causado, a heurística Prevenção de Erros não foi observada. O retrabalho poderia ter sido evitado com uma sinalização de campos obrigatórios.

Na funcionalidade "O que mais cai no ENEM" da plataforma Geekie Games, o usuário realiza diversas escolhas e entra em diversos níveis de profundidade. A interface gráfica não apresenta nenhum elemento que indique a atual localização do usuário na navegação, contrariando a primeira heurística Visibilidade do status do sistema.

O aplicativo ClassDojo, por conta da internacionalização apresenta algumas telas que falham na heurística Compatibilidade do sistema com o mundo real por utilizarem ao mesmo tempo texto em português e inglês, fazendo com que o usuário fique confuso com as funcionalidades.

Outro problema do ClassDojo causado por questões técnicas é a totalização dos alunos. Conforme o usuário adiciona alunos na turma, esse total não é atualizado, deixando os dados inconsistentes e as informações não confiáveis. Nesse ponto o sistema falha na primeira heurística: Visibilidade do status do sistema.

A funcionalidade "Criar atividade" do ClassDojo é muito bem ilustrada e minimalista. Porém, também falha na heurística de Prevenção de erros ao não sinalizar os campos obrigatórios, mostrando apenas essa informação após o usuário finalizar o cadastro.

A tela de criação de conta do Duolingo apresenta dois problemas de usabilidade relacionados entre si. Ele também falha na heurística Prevenção de erros ao não informar 
os campos requeridos, mas diferente dos demais aplicativos avaliados, as mensagens apresentadas não informam o problema para o usuário, sinalizando que o e-mail informado é inválido, mesmo o e-mail não tendo sido informado.

As demais telas avaliadas no Duolingo apresentaram uma boa interação com usuário, porém a interface de "Configuração de Conta" quebra o padrão das telas anteriores, com uma aparência simplista e com botões que não possuem aparência de botões, dando a entender que são apenas texto. Por esses detalhes, o sistema falha na heurística Consistência e Padrões e Reconhecimento ao invés de lembrança.

\subsection{Pontos Positivos}

O consenso dos avaliadores foi que as interfaces dos sistemas avaliados apresentaram um alto nível de qualidade, apesar dos pequenos problemas apresentados na Seção 5.1.

Entre os pontos positivos elencados pelos avaliadores podemos destacar as telas do Geekie Game que apresenta sempre um botão de suporte na tela, para que o usuário possa consultar a ajuda ou entrar em contato com o suporte técnico. Essa funcionalidade está de acordo com a heurística Ajuda e Documentação.

Outro bom exemplo do site Geekie Game é a tela de Aula (Figura 2) que apresenta no menu lateral uma timeline com as atividades daquela aula, fortalecendo a primeira heurística Visibilidade e status do sistema. Essa mesma timeline permite que usuário avance para uma atividade, não sendo obrigado a seguir o roteiro pré-estabelecido, em consonância com a heurística Controle do Usuário e Liberdade.

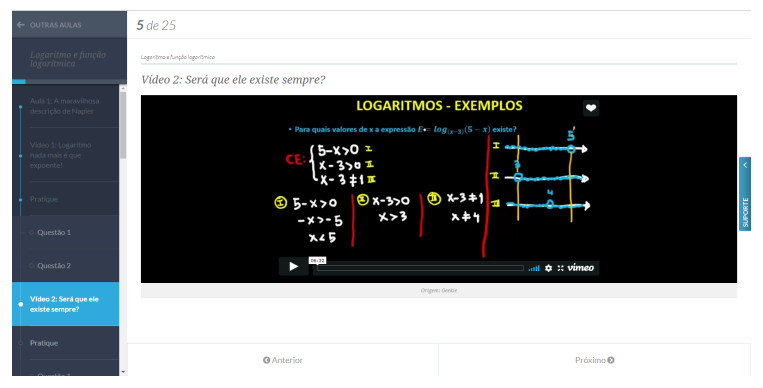

Figura 2. Tela de Aula - Geekie Games

Um destaque positivo do ClassDojo está na sua principal funcionalidade que é a atribuição de badges para alunos que evidenciam alguma competência. Nessa tela há uma clara representação dos elementos e as funcionalidades estão bem claras, podem ser acessadas diretamente com nenhuma entrada de texto. Essas qualidades estão de acordo com as heurísticas Compatibilidade do Sistema com o mundo real e Estética de Design Minimalista.

O melhor aplicativo segundo os avaliadores foi o Duolingo, ele apresenta uma interface muito agradável e consistente. Os comandos estão sempre acessíveis, o usuário consegue interagir com sistema de forma fluída, na grande maioria das vezes apenas com o mouse ou toque na tela. Das interfaces analisadas podemos destacar as telas de "Conquistas" e "Atividades" (Figura 3) que estão muito alinhadas com as heurísticas Estética e Design Minimalista, Consistência e Padrões e Visibilidade do status do sistema. 
VIII Congresso Brasileiro de Informática na Educação (CBIE 2019)

Anais do XXX Simpósio Brasileiro de Informática na Educação (SBIE 2019)

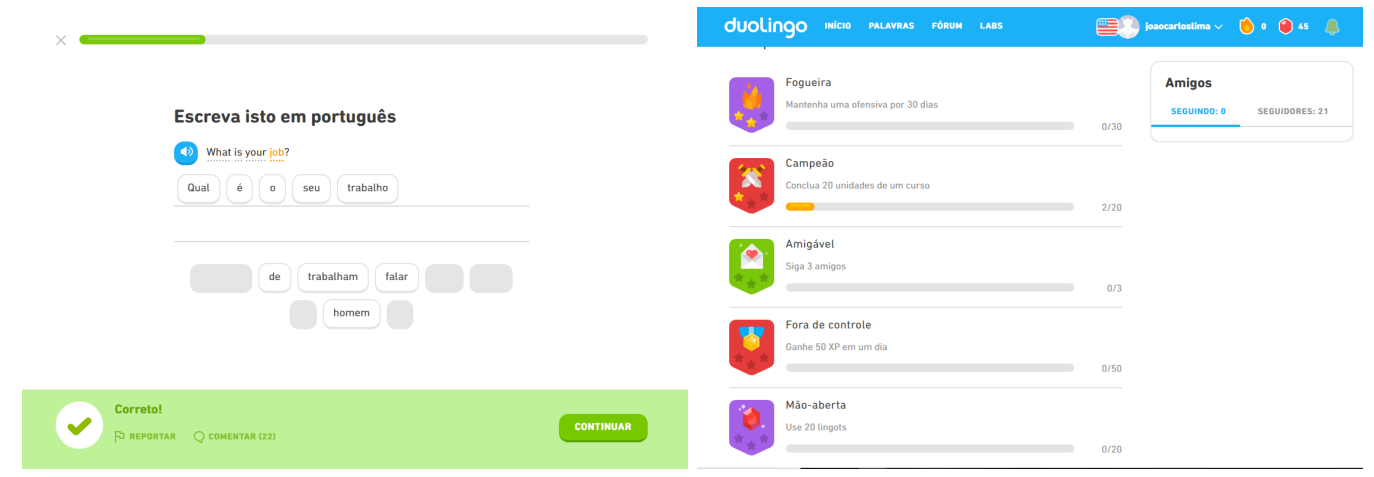

Figura 3. Telas Atividades e Conquistas - Duolingo

\subsection{Avaliações Individuais e Discussões}

Embora o objetivo da avaliação heurística seja a análise qualitativa de interfaces para identificação de problemas de usabilidade, durante a avaliação dos sistemas foram atribuídos pontos para cada uma das funcionalidades avaliadas dentro das 10 heurísticas. O resultado da avaliação está representado na Figura 4.

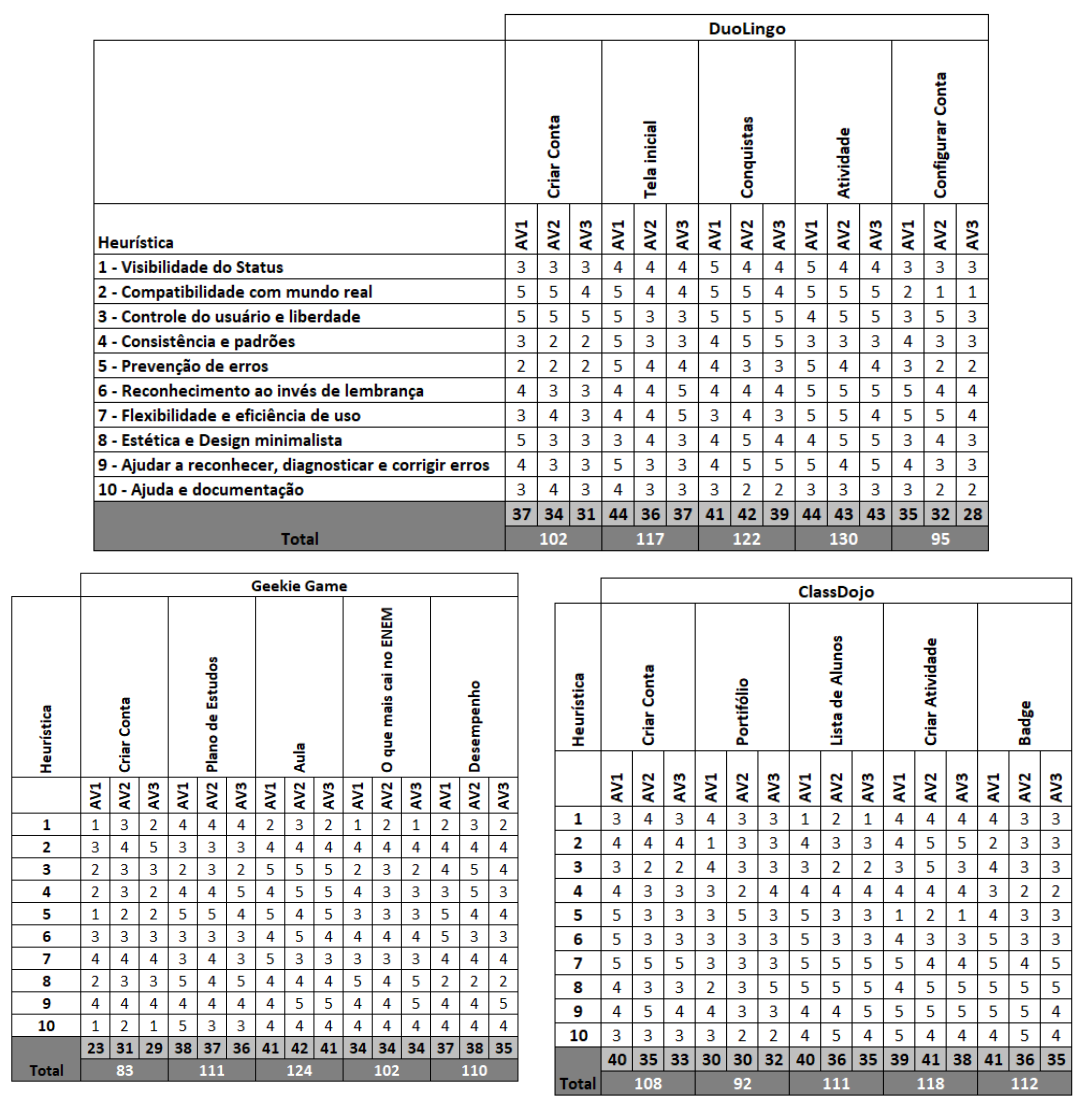

Figura 4. Avaliação Individual da Usabilidade

$\mathrm{Na}$ avaliação geral o Duolingo se saiu melhor, os outros sistemas ficaram com uma avaliação muito próxima, com uma pequena vantagem para o Geekie Game. As funcionalidades que mais pontuaram e que representam um destaque positivo foram a interface da "Atividade" do Duolingo e a "Aula" do Geekie Game. Não por acaso, essas 
VIII Congresso Brasileiro de Informática na Educação (CBIE 2019)

Anais do XXX Simpósio Brasileiro de Informática na Educação (SBIE 2019)

são as principais funcionalidades desses sistemas, o que demonstra que houve uma maior dedicação e cuidado com essas funcionalidades por parte dos analistas e desenvolvedores. Os destaques negativos ficaram para as interfaces de criação de conta do Geekie Games e ClassDojo, isso impacta diretamente na experiência do usuário, uma vez que essa dificuldade se apresenta logo no início da utilização e pode impedir o acesso do aluno ao sistema.

Em relação ao processo de avaliação proposto, o relato dos avaliadores é de que seguir o procedimento possibilitou identificar as limitações de forma ágil e clara. $\mathrm{O}$ uso da escala Likert permitiu quantificar a qualidade das funcionalidades e identificar aquelas que necessitavam de uma maior atenção.

\section{Conclusão}

Este trabalho apresentou um processo para a avaliação de interfaces de sistemas educacionais gamificados por meio das heurísticas de Nielsen. Resultados obtidos com a aplicação do processo permitiram identificar, nos sistemas avaliados, boas práticas de interface gráfica do usuário, por exemplo, padronização de interações para realização de tarefas semelhantes e feedback rápido e claro das ações do usuário. Estas práticas são fundamentais para garantir o flow das aplicações. Além disso, a utilização de símbolos, badges e linhas do tempo se mostraram bastante eficientes para ilustrar o status do sistema e a navegação pelas funcionalidades, mesmo que de forma não linear, oferecendo ao usuário a opção de liberdade de escolha para traçar seu próprio caminho de aprendizagem. Entre os principais problemas de usabilidades encontrados estão a dificuldade em criar uma conta nos sistemas, por não ser informado dos campos obrigatórios, e a dificuldade de visualizar o status do sistema, principalmente quando são visitadas hierarquias em níveis e sub-níveis. Estes problemas tendem a "quebrar"o flow de usabilidade e, por conta disso, usuários rejeitam a solução. Nesse sentido, é importante considerar que essas funcionalidades que não fazem parte do núcleo das aplicações devem ser tratadas com atenção pelos desenvolvedores.

Com base nestes resultados, este trabalho apresenta três contribuições principais: 1) um processo que permite a avaliação de sistemas educacionais gamificados; 2) lições aprendidas, empiricamente, sobre a avaliação de interfaces; e, 3) um conjunto de recomendações para a implementação de interfaces em sistemas educacionais gamificados. Essas contribuições complementam pesquisas anteriores. Esse processo pode ser uma alternativa para a metodologia aplicada por [Barbosa et al. 2014], além de atender aos trabalhos futuros sugeridos por [Souza and Souto 2015], propondo uma metodologia específica para avaliação de aplicativos gamificados. As lições aprendidas podem complementar os resultados apontados por [Zancan 2018], uma vez que os aplicativos avaliados pelos autores podem agora ter uma avaliação focada no caráter gamificado do processo educacional.

As limitações deste trabalho estão relacionadas à: (1) o número de avaliadores: o ideal é de três a cinco para uma avaliação mais precisa; e (2) a quantidade de objetos de estudo: o ideal é abordar mais sistemas para um maior entendimento sobre os pontos fortes e fracos dos sistemas educacionais gamificados (e até mesmo pagos, tais como Rosetta Stone e Feedback Panda).

Como proposta de trabalhos futuros, sugere-se a criação, aplicação e teste de 
VIII Congresso Brasileiro de Informática na Educação (CBIE 2019)

Anais do XXX Simpósio Brasileiro de Informática na Educação (SBIE 2019)

métricas heurísticas específicas para ambientes gamificados. Podendo ser adaptadas das heurísticas propostas por [Tondello et al. 2016]. Entende-se que uma avaliação focada em gamificação pode trazer ainda mais benefícios para entendimento da usabilidade desse tipo de sistema.

\section{Referências}

Alves, F. (2014). Gamification. Como Criar Experiências de Aprendizagem Engajadoras. Um Guia Completo. Do Conceito à Prática. DVS.

Barbosa, A., Silva, R., Silva, K., Santana, T., Bonfim, N., and Matos, E. (2014). Avaliação de interação do aplicativo runkeeper. IHC14 - Brazilian Symposium on Human Factors in Computing Systems, pages 27-31.

Botha, A., Herselman, M., and Ford, M. (2014). Gamification beyond badges. IST-Africa.

Csikszentmihalyi, M. (2008). Flow: The Psychology of Optimal Experience. Harper Perennial.

da Rocha, H. V. and Baranauskas, M. C. (2003). Design e Avaliação de Interfaces Humano-Computador. Núcleo de Informática Aplicada à Educação - UNICAMP.

Domingues, A., Navarrete, J., DeMarcos, L., Fernandez, L., Pages, C., and Martínez, J. J. (2013). Gamifying learning experiences: Practical implications and outcomes. Computers Education, pages 380-392.

Kapp, K. (2012). The Gamification of learning and instruction: Game-based methods and strategies for training and education. Pfeiffer Company.

Kiili, K., de Freitas, S., Arnab, S., and Lainema, T. (2012). The design principles for flow experience in educational games. Procedia Computer Science, (15):78-91.

Marache-Francisco, C. and Brangier, E. (2013). Perception of gamification: Between graphical design and persuasive design. International Conference of Design, User Experience, and Usability, 8013:558-567.

Mattar, J. (2010). Games em Educação: Como os Nativos Digitais Aprendem. Pearson.

Nielsen, J. (1994). Enhancing the explanatory power of usability heuristics. Human Factors in Computing Systems, pages 152-158.

Nielsen, J. and Landauer, T. (1993). A mathematical model of the finding of usability problems. Conference on Human Factors in Computing Systems, pages 206-213.

Nielsen, J. and Molich, R. (1990). Heuristic evaluation of user interfaces. Human Factors in Computing Systems Conference, pages 250-256.

Souza, E. R. and Souto, E. (2015). Utilização de heurísticas de jogos para avaliação de um aplicativo gamificado. SBC - SBGames, pages 666-673.

Tondello, G., Kappen, D., Mekler, E., Ganaba, M., and Nacke, L. (2016). Heuristic evaluation for gameful design. Human Factors in Computing Systems Conference, pages $315-323$.

Zancan, N. P. (2018). Avaliação heurística de jogos no ensino de química. Universidade Federal de Santa Maria. 